\title{
DIGITALCOMMONS

9-1-2015

\section{The Evolution of Tanning Needs Its Day in the Sun}

Ellen E. Quillen

Department of Genetics, Texas Biomedical Research Institute, San Antonio, TX, equillen@txbiomed.org

\section{Recommended Citation}

Quillen, Ellen E., "The Evolution of Tanning Needs Its Day in the Sun" (2015). Human Biology Open Access Pre-Prints. 109. http://digitalcommons.wayne.edu/humbiol_preprints/109 


\title{
The evolution of tanning needs its day in the sun.
}

\author{
ELLEN E. QUILLEN ${ }^{1}$
}

${ }^{1}$ Department of Genetics Texas Biomedical Research Institute P.O. Box 760549 San Antonio, Texas 78245-0549 equillen@txbiomed.org

Key words- evolution, pigmentation, tanning, facultative pigmentation

Issue- 87.4

\section{Abstract}

Variation in human pigmentation has been studied since the earliest days of biological anthropology. Categorical classifications based on references like Felix von Luschan's glass tiles were commonly used to characterize pigmentation from the late $18^{\text {th }}$ century until the introduction of machine-based automated tests in the 1950s. With this new technology, continuous measures of skin reflectance became the standard and captured a greater array of variation. There remains a significant amount of data that was only ever collected using comparison methods which may now be converted to equivalent reflectance measures (Swiatoniowski et al. 2013). Both comparison and reflectance methods have been utilized to collect pigmentation data at regions of the body exposed and unexposed to ultraviolet radiation (UVR) from the sun. This data was useful in identifying broad patterns of relationship between constitutive pigmentation (the basal amount or melanin produced by the body in the absence of UVR exposure) and facultative pigmentation (realized melanin production due to UVR exposure). Namely, those with very pale skin will develop an immediate sunburn but not tan and those with darker skin rarely have an erythemal response, but will develop increased pigmentation several days after UV exposure (Fitzpatrick 1975; Fitzpatrick 1988). (Although it must be noted here that, despite later applications, this relationship was only ever intended to be applied to individuals who were classified as "White" by dermatologists).

The substantial influence of number, frequency, duration, and intensity of UV exposures on facultative pigmentation leads to a significant amount of noise in this data. As a result, genetic studies of skin pigmentation have focused predominently on constitutive pigmentation levels with the assumption that constitutive pigmentation is predominantly driven by genetic, rather than environmental, factors. This approach has been quite successful and has identified hundreds of individual variants in a dozen or more genes contributing to differences in constitutive pigmentation within and among geographically distant populations (Parra 2007; Sturm 2009; Quillen and Shriver 2011; Sturm and Duffy 2012; Rees 
and Harding 2012). Nevertheless, this focus on the genetics of constitutive pigmentation means something important is lost in our understanding of how human skin interacts with the environment. This paper makes the argument that the pigmentation induced by solar exposure is an evolutionarily relevant phenotype and establishes the hypothesis that persistence of increased facultative pigmentation in the Americas could be an example of convergent evolution with the greater constitutive pigmentation seen in high-UVR populations of Africa and Asia.

\section{Photoprotection Appears among the Strongest Forces of Selection in Human Populations}

Large studies of constitutive skin reflectance data, predominantly from populations in Africa, Europe, Asia, and Oceania, led to the identification of a link between latitude and pigmentation that was later demonstrated to reflect a stronger correlation between increased ultraviolet radiation (UVR) and darker skin color (Walter 1971; Byard 1981; Robins 1991; Frisancho 1993; Relethford 1997; Jablonski and Chaplin 2000). Evidence for the adaptive nature of this relationship comes from genomic scans for selection in Europeans, East Asians, and South Asians, which indicate that genetic variation at pigmentation genes has been shaped by some of the strongest intervals of selection to leave their mark on human genomes (Akey et al. 2002; Voight et al. 2006; Sabeti et al. 2007; Stokowski et al. 2007; Lao et al. 2007; Johansson and Gyllesnsten 2008; Myles et al. 2008; Pickrell et al. 2009; Hancock et al. 2011; Sturm and Duffy 2012).

A variety of hypotheses have been considered to explain why skin pigmentation would have such a large effect on fitness. The best supported are protection from photolysis of folic acid (Branda and Eaton 1978; Blount 1997; Fleming 1998) and from sunburn and skin cancer (Weinstock et al. 1989; Lookingbill et al. 1995; Greaves 2014). These hypotheses are not mutually exclusive, and predominantly relate to the photoprotective effect of high levels of epidermal eumelanin, the brown-black pigment responsible for most variation in human skin color. An alternative hypothesis contends that xeric stress in a hot dry climate could also favor the thicker, acidified stratum corneum that is a byproduct of increased melanin (Elias et al. 2010). However, this hypothesis is inconsistent with the many regions of the world where individuals with extremely dark skin pigmentation occupy areas of very high humidity, e.g. equatorial west Africa, new Guinea, and the tropical regions of northern Australia. Independent of the precise evolutionary mechanisms, the evidence for selection in favor of darker skin in highUVR regions - generally found closer to the equator - is strong.

Ground-level UVR varies world-wide with altitude, density of vegetation, and cloud-cover resulting in inexact UVR correlations between the same latitudes in the Eastern hemisphere, where the majority of research has been done, and the Western hemisphere. Unfortunately, genetic research on skin color in the Americas 
has been limited by scarcity of data on Indigenous American populations using modern reflectance methods. However, it is evident even from Renato Biasutti's (1959) categorical data on skin color that indigenous populations living in tropical and subtropical regions of the Americas have low levels of constitutive pigmentation relative to populations living at similar latitudes in Africa and Melanesia (Barsh 2003), even when the lower UVR levels in tropical regions of the Americas are considered (Jablonski and Chaplin 2000).

There are several possible explanations for this discrepancy. Indigenous American populations have undergone at least one population bottleneck, reducing genetic diversity and potentially eliminating some variation at pigmentation loci (Hey 2005) which may have already been reduced by selection favoring lighter pigmentation in ancestral populations. This demographic explanation is a null hypothesis against which all other hypothesis should be measured. Although humans have only been occupying high-UVR regions of the Americas for approximately 10,000 years (Cooke 1998), this time span was shown to be sufficient for substantial changes to occur in the skin pigmentation of several Old World populations which have also undergone recent, though milder, bottlenecks (Lao et al. 2007; Stokowski et al. 2007). Additionally, the Americas were populated relatively late, meaning mechanisms of cultural buffering - particularly clothing that would mitigate some UVR exposure - were already in use.

\section{Facultative Pigmentation has Photoprotective Ability}

Considering established examples of rapid evolution at pigmentation genes in European, East Asian, and South Asian populations and the known deleterious effects of low levels of melanin in individuals exposed to intense UVR, the relatively light constitutive pigmentation in Indigenous Americans is anomalous. Of the pigmentation genes that appear to have undergone selection in the ancestors of contemporary Indigenous Americans, only two have even small effects on the constitutive pigmentation of modern admixed Indigenous American and European populations (Quillen et al. 2011). That research, like most on skin pigmentation, focused on constitutive pigmentation which has been presumed to be more directly genetically determined, and therefore more susceptible to selection, than facultative pigmentation which is a response to environmental exposure. However, this assumption discounts the reality that facultative pigmentation is the true interface between humans and the environment because skin begins to respond nearly instantaneously to UVR.

Facultative melanogenesis (tanning) is a photoprotective response triggered by DNA damage and repair following UVR exposure which can result in a 7-10 fold increase in visible skin pigmentation (Sheehan et al. 1998; Gilchrest and Eller 1999). This significant variability in pigmentation has been suggested as a major component of photoprotection previously (Wagner et al. 2002; Tadokoro et al. 
2003; Halder and Ara 2003; Tadokoro et al. 2005; Brenner and Hearing 2008; Wolber et al. 2008; Miller et al. 2008; Coelho et al. 2009a). Like constitutive pigmentation, this tanning response is variable and there is no reason to assume it, like other types of phenotypic plasticity, is not also partially genetically regulated. Indeed, evidence of inter-population variation in tanning abilities (Wagner et al. 2002) as well as genes association with variation in tanning phenotypes in European (Nan et al. 2009), Mongolian (Paik et al. 2011), and Korean (Kwak et al. 2015) populations have been identified. (It should again be noted that the majority of previous studies in this area have simply compared exposed to unexposed regions of the body as a proxy for tanning capacity. This fails to account for variation in exposure and can lead to noise in the data and false negative results.)

The features of melanin that prevent cellular and DNA damage - broad UVR absorption (Anderson and Parrish 1981; Kollias and Baqer 1987) and distribution near the surface of the skin (Kobayashi et al. 1998) - are the same for both constitutive pigmentation and UVB-induced facultative pigmentation. It follows, then that one hypothesis for the divergence of Indigenous American skin pigmentation from the expected values is that facultative pigmentation, rather than constitutive pigmentation, is the primary means of avoiding UVR-induced damage. While dermatologists have long noted - and in some cases assumed - that individuals with darker skin pigmentation are more like to tan than fairer individuals, less is known about inter-population differences. The small amount of available literature suggests that East Asian individuals show a stronger and more sustained melanogenic response to UVR exposure than Europeans.

A similar photoadaptive response in Indigenous American populations would mitigate the selective pressures favoring darker constitutive pigmentation in a high-UVR environment and explain the observed discrepancy in pigmentation between Eastern and Western hemisphere populations residing at the same latitude. From an evolutionary perspective, pigmentation gained due to tanning is as adaptive as darker constitutive pigmentation. Since evolution works only on the phenotype, there is no reason to expect that darker constitutive pigmentation would evolve more commonly than a strong tanning response. Additionally, tanning capability may be adaptive in and of itself in the subtropical regions where seasonal variation in UVR dips below the threshold needed for cutaneous vitamin D production in dark skin for more than three months out of the year (Chaplin and Jablonski 2009). Such phenotypic plasticity would enable individuals to become progressively lighter in the winter months when reserves of vitamin D were depleted or to oscillate between less melanin during extended periods in forest climates where UVR is lower and increased melanin in response to exposure to elevated UVR in more arid climates.

The limitation of facultative melanogenesis in increasing fitness is the significant damage that may occur due to UVR before melanogenesis occurs. The 
balance between initial damage due to relatively light constitutive pigmentation and photoprotective facultative pigmentation would be shifted by two factors: (1) the amount of UV exposure necessary to induced facultative melanogenesis (MMD, the minimum melanogenic dose); and (2) the persistence of the acquired, protective melanin to reduce future damage. Epidermal exposure to UVR induces separate but related reactions which vary among individuals -immediate pigment darkening occurs within minutes of UV exposure (Hönigsmann et al. 1986; Routaboul et al. 1999), persistent pigment darkening develops over several hours and persists for days (Moyal et al. 2000; Moyal et al. 2006), and delayed pigment darkening which can take more than a day to appear and persists from several weeks to six months or more (Tadokoro et al. 2003; Tadokoro et al. 2005; Young 2006). Importantly, research indicates that only tanning related to UVB, not UVA, radiation is photoprotective (Miyamura et al. 2011, Coelho et al 2015). UVB, which varies seasonally at latitudes greater than $45^{\circ}$, elicits persistent changes in pigmentation via upregulation of pigmentation pathway genes while longer-wavelength, lower energy UVA is linked to immediate, short-term changes in skin pigmentation by oxidation and redistribution of existing melanin and melanin-precursors (Choi et al. 2010). UVA and UVB trigger expression of overlapping but partially independent genetic pathways (Brenner et al. 2009). Based on these observations, facultative pigmentation is most likely to be protective, and therefore evolutionarily relevant, in regions with variable UVR due to seasonal differences or changes in cloud or tree cover.

The molecular mechanisms for the longer-term tanning response relies predominantly on the stimulation of the cyclic-AMP pathway (Chakraborty et al. 1996) which upregulates the production of the melanocyte stimulating hormone (MSH) and the melanocortin-1 receptor (MC1R) (Funasaka et al. 1998). Changes in protein concentrations can persist over the course of several weeks following UV exposure and occur in a well-documented pattern (Coelho et al. 2009b). The net effect of these changes is increased melanin production in the melanocyte and transfer to keratinocytes closer to the epidermal surface (Gilchrest and Eller 1999), a phenomenon generally more extreme in individuals with darker constitutive pigmentation (Scheibner et al. 1986; Stierner et al. 1989). Although the majority of research has focused primarily on pigmentation, it should be noted that repeated, acute, erythema-inducing UV exposure increases the thickness of the epidermis and stratum corneum which also reduces transmission of UV through the skin (Brenner and Hearing 2008).

Population-Level Variability in Sensitivity and Persistence of UVR Response Both immediate and short-term onset tanning responses vary within and among human populations (Kalla 1974; Coelho et al. 2009a). One of the few studies to compare multiple European-American, Hispanic, and East Asian individuals 
collected under a single protocol identified substantial differences among these groups in average response to UVR (Wagner et al. 2002). This work considered the minimum amount of UVR necessary to cause a reddening of the skin (minimum erythemal dose, MED) or induce a melanogenic reaction (minimum melanogenic dose, MMD) as well as the dose-response curves for both (erythemal dose response, EDR, and melanogenic dose response, MDR). Self-identified Hispanic and East Asian participants on average showed a stronger tanning response than European Americans for all measures. However, this research only considered facultative pigmentation at 24 hours and 7 days, meaning that persistence of facultative pigmentation could not be evaluated.

Few investigations have considered variation in delayed-onset, persistent facultative pigmentation, and all have been underpowered to look at populationlevel differences. From an evolutionary perspective, this type of facultative pigmentation may be the most important as it extends the period of photoprotection from a single UVR exposure. Investigations of persistent changes in pigmentation come predominantly from dermatological research performed in East Asia. Among Korean patients undergoing weekly UVB phototherapy for psoriasis, maximum pigmentation was reached after five weeks of sub-MED exposures (Choe et al. 2002) and required 10 weeks to 10 months to return to pre-exposure levels (Jo et al. 2006; Suh et al. 2007; Kwon et al. 2010). The difference in tanning response between European and East Asian individuals has been noted frequently in the dermatological literature, often with the conclusion that the application of the Fitzpatrick scale (Fitzpatrick 1975), which in common practice conflates constitutive pigmentation with tanning response, is not applicable to many East Asian populations. While levels of constitutive eumelanin and initial erythemal response may be similar between East Asians and Western Europeans, the persistent facultative pigmentation response appears substantially stronger, particularly in Chinese and Japanese populations (Kawada 1986; Satoh and Kawada 1986; Kawada et al. 1993; Liao et al. 1995; Stanford et al. 1996; Youn et al. 1997; Wee et al. 1997; Park et al. 1998; Liu et al. 2006). This conclusion is preliminary as it is based exclusively on small studies of phototype or repeated medical exposures to UVR. No large-scale studies have considered persistent facultative pigmentation in samples of known ancestry and the distribution of neither intensity nor persistence of tanning response is known.

Persistent facultative pigmentation has not been documented in Central Asian populations who are a better proxy for the ancestors of Indigenous Americans than East Asians, although there is no evidence to suggest that it does not occur. The only study to consider facultative pigmentation in a large Central Asian sample determined tanning response by comparing exposed to unexposed regions of the body within an isolated Mongolian nomadic group (Paik et al. 2011). This does not take into consideration amount of exposure or persistence of facultative 
pigmentation. Unfortunately, there exists little consistently collected data on facultative pigmentation as it is heavily influenced by seasonality of UVR exposure, age as tanning capacity changes over the life course, and cultural differences in patterns of exposure and norms of body cover. Measuring tanning response in a controlled setting is the best way to consider this question.

Procaccini et al. (2007) considered the relationship between MED and duration of pigmentation (up to 16 days) in 50 individuals of Fitzpatrick phototypes II (fair skin, burns easily, tans poorly) through IV (light brown skin, burns minimally, tans easily). Mean differences in duration were identified among the phototypes with individuals of phototype II more like to return to constitutive pigmentation within 16 days. However, substantial variation was found within each group. In a longer-term study of 60 individuals with a broader range of constitutive pigmentation, Coelho et al. (2009a) found that some individuals exhibited facultative pigmentation up to nine months after exposure to UVR three times their MED. In this study, greater MED and greater MMD, but not constitutive pigmentation levels, were associated with an increased likelihood of this longlasting pigmentation, illustrating the partial independence of the constitutive pigmentation and facultative pigmentation pathways. While this study included some individuals with putative Indigenous American ancestry, biogeographic ancestry (BGA) was not measured and there were insufficient participants to analyze differences among self-reported racial or ethnic groups.

Tanning phenotypes have very rarely been considered in Indigenous American populations outside of research into melanoma. Limited evidence for differences in tanning response between Indigenous American and European individuals comes from studies of phototype in Colombia (Sanclemente et al. 2008). This research found a small association between MED and phototype and, as in East Asian populations, an overall difference in photosensitivity between Europeans and admixed Colombians. While the associations between MED, phototype, and tanning are complex, this data suggests differences in tanning ability may exist between Indigenous Americans and Old World populations that merit further investigation. If present, persistent facultative pigmentation in Indigenous Americans may be a shared phenotype with Asian populations and a "preadaptation" to increased UVR in the tropical and sub-tropical regions of the Americas.

As expected based on the phenotypic pattern, the genetic variants thus far implicated in variation in tanning response partially overlap with those contributing to constitutive pigmentation. Canonical pigmentation genes where variants are associated with differences in tanning capacity include WNTI and SILV/Pmell7 (Paik et al. 2011) in a Mongolian population and SLC45A2/MATP, IRF4, TYR, $O C A 2, H E R C 2, P P A R G C 1 B, M I T F$ and MC1R in broadly-collected European and European-American populations (Nan et al. 2009; Shoag et al. 2012; Zhang et al. 
2013). Additionally, these association studies identified variants in unexpected loci including GRM6 and ATF1 (Paik et al. 2011) and LOC401937 (Nan et al. 2009). Not previously considered a pigmentation candidate gene, variation in $W W O X$ within Korean women is related to tanning capacity and regulates the activity of tyrosinase in human melanocytes (Kwak et al. 2015). As more is known about the genes regulating tanning, it is likely that many genes but not all individual mutations will influence both types of pigmentation. Thus far, no studies have considered admixed populations which would allow the identification of genes contributing to between population differences in tanning response.

\section{Conclusion}

Drawn from many sources, this body of evidence suggests that facultative pigmentation may play a significant role in the adaptation of populations of the Americas to increased UVR in tropical and subtropical regions. The time has come for an increased focus on the evolutionary genetics of facultative pigmentation not only in the Americas, but more broadly. The study of human pigmentation is fundamental to anthropology because it represents an enormously variable phenotype present in all humans. A prime example of the interactions between humans and our highly variable environment, skin pigmentation has been shaped and reshaped by the forces of evolution over the past several thousand years of the hominid lineage. There is substantial ongoing debate in the anthropological literature regarding the proximate evolutionary forces (selection vs. drift) (Robins 2009) as well as the nature of selective pressures acting on skin (Black et al. 1987; Chaplin and Jablonski 2009). Resolution of this debate rests on a full understanding of what types of pigmentation contribute to variation in modern humans. An exclusive focus on constitutive pigmentation unduly limits our understanding of skin pigmentation and how humans have adapted to diverse environments. The inclusion of facultative pigmentation alongside constitutive pigmentation could be the missing piece in anthropologists' understanding of the relationship between UVR and skin color.

Acknowledgements: The author would like to thank Nina Jablonski and Mark Shriver for their many discussions about the evolution and genetics of facultative pigmentation, without which this manuscript would not have been developed.

Received 4 January 2016; revision uploaded 22 April 2016. 


\section{Literature cited}

Akey, J. M., G. Zhang, K. Zhang et al. 2002. Interrogating a high-density SNP map for signatures of natural selection. Genome res., 12(12), 1805-14.

Anderson, R., J. A. Parrish. 1981. The optics of human skin. J. Invest. Dermatol., 77(1), 13-19.

Barsh, G. S. 2003. What controls variation in human skin color? PLoS Biol., 1(1), E27.

Black, W. C., R. Goldhahn, C. Wiggins. 1987. Melanoma within a southwestern Hispanic population. Arch. Dermatol., 123(10), 1331-1334.

Blount, B. C.1997. Folate deficiency causes uracil misincorporation into human DNA and chromosome breakage: Implications for cancer and neuronal damage. P. Natl. Acad. Sci. USA, 94(7), 3290-3295.

Branda, R., J. Eaton. 1978. Skin color and nutrient photolysis: an evolutionary hypothesis. Science, 201(4356), 625-626.

Brenner, M., S. G. Coelho, J. Z. Beer et al. 2009. Long-lasting molecular changes in human skin after repetitive in situ UV irradiation. J. Invest. Dermatol., 129(4), 1002-11.

Brenner, M., V. J. Hearing. 2008. The protective role of melanin against UV damage in human skin. Photochem. Photobiol., 84(3), 539-549.

Byard, P. J. 1981. Quantitative genetics of human skin color. Am. J. Phys. Anthropol., 24(S2), 123-137.

Chakraborty, K., Y. Funasaka, A. Slominski et al. 1996. Production and release of proopiomelanocortin (POMC) derived peptides by human melanocytes and keratinocytes in culture: regulation by ultraviolet B. Biochim. Biophys. Acta, 1313(2), 130-8.

Chaplin, G., N. G. Jablonski. 2009. Vitamin D and the evolution of human depigmentation. Am. J. Phys. Anthropol., 139(4), 451-61. 
Choe, Y. B., J. H. Rim, J. I. Youn. 2002. Quantitative assessment of narrow-band UVB induced tanning during phototherapy in Korea. Photodermatol. Photo., 18(3), 127-30.

Choi, W., Y. Miyamura, R. Wolber et al. 2010. Regulation of human skin pigmentation in situ by repetitive UV exposure: molecular characterization of responses to UVA and/or UVB. J. Invest. Dermatol., 130(6), 1685-96.

Coelho, S. G., W. Choi, M. Brenner et al. 2009a. Short- and long-term effects of UV radiation on the pigmentation of human skin. J. Invest. Dermatol. . Symposium Proceedings, 14(1), 32-5.

Coelho, S. G., Y. Zhou, H. F. Bushar et al. 2009b. Long-lasting pigmentation of human skin, a new look at an overlooked response to UV. Pigm. Cell Melanoma R., 22(2), 238-41.

Coelho, S. G., L. Yin, C. Smuda et al. 2015, Photobiological implications of melanin photoprotection after UVB-induced tanning of human skin but not UVA-induced tanning. Pigm. Cell Melanoma R., 28: 210-216.

Cooke, R. 1998. Human settlement of Central America and Northernmost South America (14,000-8000 BP). Quatern. Int., 49-50, 177-190.

Elias, P., G. Menon, B. Wetzel et al. 2010. Barrier requirements as the evolutionary "driver" of epidermal pigmentation in humans. Am. J. Hum. Biol., 22(4), 526-537.

Fitzpatrick, T. 1988. The validity and practicality of sun-reactive skin types I through VI. Arch. Dermatol., 124(6), 869-71.

Fitzpatrick, T. B. 1975. Soleil et peau. J. Med. Esthet., 2(7), 33-34.

Fleming, A. 1998. Embryonic Folate Metabolism and Mouse Neural Tube Defects. Science, 280(5372), 2107-2109.

Frisancho, A. R. 1993. Human adaptation and accommodation. Ann Arbor, MI: University of Michigan Press.

Funasaka, Y., A. K. Chakraborty, Y. Hayashi et al. 1998. Modulation of melanocyte-stimulating hormone receptor expression on normal human melanocytes: evidence for a regulatory role of ultraviolet $\mathrm{B}$, interleukin-1 $\alpha$, 
interleukin-1 $\beta$, endothelin-1 and tumour necrosis factor- $\alpha$. Brit. J. Dermatol., $139,216-224$.

Gilchrest, B., M. S. Eller. 1999. DNA photodamage stimulates melanogenesis and other photoprotective responses. J. Invest. Dermatol. . Symposium Proceedings, 4(1), 35-40.

Greaves, M. 2014. Was skin cancer a selective force for black pigmentation in early hominin evolution? Proc. Biol. Sci., 281, 20132955.

Halder, R. M., C. J. Ara. 2003. Skin cancer and photoaging in ethnic skin. Dermatol. Clin., 21(4), 725-732.

Hancock, A. M., D. B. Witonsky, G. Alkorta-Aranburu et al. 2011. Adaptations to climate-mediated selective pressures in humans. PLoS Genet., 7(4), e1001375.

Hey, J. 2005. On the number of New World founders: a population genetic portrait of the peopling of the Americas. PLoS Biol., 3(6), e193.

Hönigsmann, H., G. Schuler, W. Aberer et al. 1986. Immediate pigment darkening phenomenon. A reevaluation of its mechanisms. J. Invest. Dermatol., 87(5), 648-652.

Jablonski, N. G., G. Chaplin. 2000. The evolution of human skin coloration. $J$. Hum. Evol., 39(1), 57-106.

Jo, S. J., H. S. Yoon, S. M. Woo et al. 2006. Time course of tanning induced by narrow-band UVB phototherapy in Korean psoriasis patients.

Photodermatol. Photo., 22(4), 193-199.

Johansson, A., U. Gyllesnsten. 2008. Identification of local selective sweeps in human populations since the exodus from Africa. Hereditas, 145, 126-137.

Kalla, A. 1974. Human skin pigmentation, its genetics and variation. Hum. Genet., 300, 289-300.

Kawada, A. 1986. UVB-induced erythema, delayed tanning, and UVA-induced immediate tanning in Japanese skin. Photodermatol. Photo., 3, 327-333. 
Kawada, A., T. Noda, M. Hiroma et al. 1993. The relationship of sun protection factor to minimal erythema dose, Japanese skin type, and skin color. $J$. Dermatol. Sci., 20, 514-516.

Kobayashi, N., A. Nakagawa, T. Muramatsu et al. 1998. Supranuclear melanin caps reduce ultraviolet induced DNA photoproducts in human epidermis. $J$. Invest. Dermatol., 110(5), 806-810.

Kollias, N., A. H. Baqer. 1987. Absorption mechanisms of human melanin in the visible, 400-720 nm. J. Invest. Dermatol., 89(4), 384-388.

Kwak, T.-J., Y.-H. Chang, Y.-A. Shin et al. 2015. Identification of a possible susceptibility locus for UVB-induced skin tanning phenotype in Korean females using genome-wide association study. Exp. Dermatol., 12:942-6.

Kwon, I. H., S. M. Woo, J. W. Choi et al. 2010. Recovery from tanning induced by narrow-band UVB phototherapy in brown-skinned individuals with psoriasis: twelve-month follow-up. J. Dermatol. Sci., 57(1), 12-8.

Lao, O., J. M. de Gruijter, K. van Duijn et al. 2007. Signatures of positive selection in genes associated with human skin pigmentation as revealed from analyses of single nucleotide polymorphisms. Ann. Hum. Genet., 71(Pt 3), 354-69.

Liao, K., C. Yan, Y. Wang. 1995. The measurements of sun reactive skin types and its clinical relevance. Chinese Journal of Dermatology, 28, 287-289.

Liu, W., W. Lai, X. M. Wang et al. 2006. Skin phototyping in a Chinese female population: analysis of four hundred and four cases from four major cities of China. Photodermatol. Photo., 22(4), 184-8.

Lookingbill, D. P., G. L. Lookingbill, B. Leppard. 1995. Actinic damage and skin cancer in albinos in northern Tanzania: findings in 164 patients enrolled in an outreach skin care program. J. Am. Acad. Dermatol., 32(4), 653-8.

Miller, S. A, S. G. Coelho, B. Z. Zmudzka et al. 2008. Dynamics of pigmentation induction by repeated ultraviolet exposures: dose, dose interval and ultraviolet spectrum dependence. The Brit. J. Dermatol., 159(4), 921-30. 
Miyamura, Y., S. G. Coelho, K. Schlenz et al. 2011. The deceptive nature of UVA tanning versus the modest protective effects of UVB tanning on human skin. Pigm. Cell Melanoma R., 24(1), 136-47.

Moyal, D., A. Chardon, N. Kollias. 2000. Determination of UVA protection factors using the persistent pigment darkening (PPD) as the end point. Photodermatol. Photo., 16(6), 245-249.

Moyal, D., K. Wichrowski, C. Tricaud. 2006. In vivo persistent pigment darkening method: a demonstration of the reproducibility of the UVA protection factors results at several testing laboratories. Photodermatol. Photo., 22, 124-128.

Myles, S., K. Tang, M. Somel et al. 2008. Identification and analysis of genomic regions with large between-population differentiation in humans. Ann. Hum. Genet., 72(Pt 1), 99-110.

Nan, H., P. Kraft, A. A. Qureshi et al. 2009. Genome-wide association study of tanning phenotype in a population of European ancestry.. J. Invest. Dermatol., 129(9), 2250-2257.

Paik, S. H., H.-J. Kim, S. Lee et al. 2011. Linkage and association scan for tanning ability in an isolated Mongolian population. BMB Reports, 44(11), $741-6$.

Park, S., D. Suh, J. Youn. 1998. Reliability of self-assessment in determining skin phototype for Korean brown skin. Photodermatol. Photo., 14, 160-163.

Parra, E. J. 2007. Human pigmentation variation: evolution, genetic basis, and implications for public health. Yearb. Phys. Anthropol., 50, 85-105.

Pickrell, J. K., G. Coop, J. Novembre et al. 2009. Signals of recent positive selection in a worldwide sample of human populations. Genome res., 19(5), $826-37$.

Procaccini, E. M., A. Napolitano, L. Panzella et al. 2007. Long-lasting pigmentation more than its intensity is a reliable indicator of skin sun resistance. Dermatology (Basel, Switzerland), 215(3), 173-9. 
Quillen, E. E., M. Bauchet, A. W. Bigham et al. 2012. OPRM1 and EGFR contribute to skin pigmentation differences between Indigenous Americans and Europeans. Hum. Genet. 131(7):1073-80.

Quillen, E. E., M. D. Shriver. 2011. Unpacking human evolution to find the genetic determinants of human skin pigmentation. J. Invest. Dermatol., 131(E1), E5-7.

Rees, J. L., R. M. Harding. 2012. Understanding the evolution of human pigmentation: recent contributions from population genetics. J. Invest. Dermatol., 132(3 Pt 2), 846-53.

Relethford, J. H. 1997. Hemispheric difference in human skin color. Am. J. Phys. Anthropol., 104(4), 449-57.

Robins, A. 1991. Biological perspectives on human pigmentation. Cambridge: Cambridge University Press.

Robins, A. H. 2009. The evolution of light skin color: role of vitamin D disputed. Am. J. Phys. Anthropol., 139(4), 447-50.

Routaboul, C., A. Denis, A. Vinche. 1999. Immediate pigment darkening: description, kinetic and biological function. Eur. J. Dermatol., 9(2), 95-99.

Sabeti, P. C., P. Varilly, B. Fry et al. 2007. Genome-wide detection and characterization of positive selection in human populations. Nature, 449(7164), 913-8.

Sanclemente, G., J.-F. Zapata, J.-J. García et al. 2008. Lack of correlation between minimal erythema dose and skin phototype in a Colombian scholar population. Skin Res. Technol., 14(4), 403-9.

Satoh, Y., A. Kawada. 1986. Action spectrum for melanin pigmentation to ultraviolet light, and Japanese skin typing. In T. Fitzpatrick, M. Wick, K. Toda (Eds.), Brown melanoderma. Biology and disease of epidermal pigmentation. (pp. 87-95). Tokyo: University of Tokyo Press.

Scheibner, A., D. Hollis, W. McCarthy et al. 1986. Effects of sunlight exposure on Langerhans cells and melanocytes in human epidermis.

Photodermatology, 3(1), 15-25.

Pre-print version. Visit http://digitalcommons.wayne.edu/humbiol/ after publication to acquire the final version. 
Sheehan, J. M., C. S. Potten, A. R. Young. 1998. Tanning in Human Skin Types II and III Offers Modest Photoprotection Against Erythema. Photochem. Photobiol., 68, 588-592.

Shoag, J., R. Haq, M. Zhang et al. 2012. PGC-1 Coactivators Regulate MITF and the Tanning Response. Mol. Cell, 1-13.

Stanford, D., K. Georgouras, E. Sullivan et al. 1996. Skin phototyping in Asian Australians. Australias. J. Dermatol., 37(Suppl 1), S36-38.

Stierner, U., I. Rosdahl, A. Augustsson et al. 1989. UVB Irradiation Induces Melanocyte Increase in Both Exposed and Shielded Human Skin. J. Invest. Dermatol., 92, 561-564.

Stokowski, R. P., P. V. K. Pant, T. Dadd et al. 2007. A genomewide association study of skin pigmentation in a South Asian population. Am. J. Hum. Genet., 81(6), 1119-32.

Sturm, R. A. 2009. Molecular genetics of human pigmentation diversity. Hum. Mol. Genet., 18(R1), R9-17.

Sturm, R. A, D. L. Duffy. 2012. Human pigmentation genes under environmental selection. Genome Biol., 13(9), 248.

Suh, K.-S., H.-J. Roh, S.-Y. Choi et al. 2007. Long-term evaluation of erythema and pigmentation induced by ultraviolet radiations of different wavelengths. Skin Res. Technol., 13(2), 154-61.

Swiatoniowski, A. K., E. E. Quillen, M. D. Shriver et al. 2013. Technical Note: Comparing von Luschan skin color tiles and modern spectrophotometry for measuring human skin pigmentation. Am. J. Phys. Anthropol., 151(2), 325330.

Tadokoro, T., N. Kobayashi, B. Z. Zmudzka et al. 2003. UV-induced DNA damage and melanin content in human skin differing in racial/ethnic origin. Faseb. J., 17(9), 1177-9.

Tadokoro, T., Y. Yamaguchi, J. Batzer et al. 2005. Mechanisms of skin tanning in different racial/ethnic groups in response to ultraviolet radiation. J. Invest. Dermatol., 124(6), 1326-32. 
Voight, B. F., S. Kudaravalli, X. Wen et al. 2006. A map of recent positive selection in the human genome. PLoS Biol., 4(3), e72.

Wagner, J. K., C. Jovel, H. L. Norton et al. 2002. Comparing quantitative measures of erythema, pigmentation and skin response using reflectometry. Pigm. Cell Res., 15(5), 379-84.

Wagner, J. K., E. J. Parra, E. J., L. H. Norton et al. 2002. Skin responses to ultraviolet radiation: effects of constitutive pigmentation, sex, and ancestry. Pigm. Cell Res., 15(5), 385-90.

Walter, H. 1971. Remarks on the environmental adaptation of man. Humangenetik, 13(2), 85-97.

Wee, L., T. Chong, D. Quee. 1997. Assessment of skin types, skin colors and cutaneous responses to ultraviolet radiation in an Asian population.

Photodermatol. Photo., 13, 169-172.

Weinstock, M. A., G. A. Colditz, W. C. Willett et al. 1989. Nonfamilial Cutaneous Melanoma Incidence in Women Associated With Sun Exposure Before 20 Years of Age. Pediatrics, 84(2), 199-204.

Wolber, R., K. Schlenz, K. Wakamatsu et al. 2008. Pigmentation effects of solarsimulated radiation as compared with UVA and UVB radiation. Pigm. Cell Melanoma R., 21(4), 487-491.

Youn, J., J. Oh, B. Kim. 1997. Relationship between skin phototype and MED in Korean, brown skin. Photodermatol. Photo. 1, 13, 208-211.

Young, A. 2006. Acute effects of UVR on human eyes and skin. Prog. Biophy Mol. Biol., 92(1), 80-85.

Zhang, M., F. Song, L. Liang et al. 2013. Genome-wide association studies identify several new loci associated with pigmentation traits and skin cancer risk in European Americans. Hum. Mol. Genet., 22(14), 2948-2959. 